\title{
Numerical simulation of dispersion around a cubic building: characterization of wind-induced pressure coefficients on cube using Standard K- $\varepsilon$ Model
}

\author{
Ali R.Hayajneh ${ }^{1 *}$ \\ Institute of Nuclear Physics and Engineering, National Research Nuclear University \\ MEPhI (Moscow Engineering Physics Institute) \\ 115409, Russian Federation, Moscow, Kashirskoe shosse, 31 \\ Phone: +7 964 525-6196 \\ *E-mail of the corresponding author: arhayajneh@gmail.com
}

\begin{abstract}
:
The aim of the work is to determine the total pressure coefficient $\left(C_{p}\right)$, the behavior of pressure and pressure coefficient $C_{p}$ near the walls of cube (roof and floor of cube) to study the effect of $C_{p}$ on the behavior of flow and reverse flow on the top of cube due to wind tunnel, which allows the pressure due to the wind at the cube faces (negative pressure $\left(C_{p}<0\right)$ or overpressure $\left(C_{p}>0\right)$ compared to the static pressure (atmospheric pressure)) to be quantified. This coefficient depends on the wind velocity $(\mathbf{W})$ upstream the cube (velocity in the wind tunnel), on the geometry of obstacle (which is cube used here) and on the attack angle of wind.
\end{abstract}

Keywords: K- $\varepsilon$ Model, Pressure Coefficient, Wind (flow), CFD, Fluent.

DOI: $10.7176 / \mathrm{MTM} / 9-2-06$

\section{Introduction:}

CFD (RANS)simulations results of this work were compared with The experimental wind-induced mean pressure coefficients from Martinuzzi et al. (1993) [3] on "the flow around surface-mounted, prismatic obstacles placed in a fully developed channel flow" and Castro and A. G. Robins (1977, England) [8] on their study "The flow around a surface-mounted cube in uniform and turbulent streams", in order to validate the CFD modeling approach, which could be used for predicting pressure coefficients on other geometries. One wind directions was considered (Zero degree) in the direction of horizontal axis. All of the simulations were performed with the commercial ANSYS FLUENT 14.5 software (www.ansys.com) [5] using Standard K- $\varepsilon$ Model.

It should be noted that the modeling is consistent to the Recommendation of Martinuzzi (1993 , 1997) [3] and Mohd ARIFF , 2009 [2]. Ricciardi, Thomas, Sandrine (2015, France) [9] based on their study : "Experimental and numerical characterization of wind-induced pressure coefficients on nuclear buildings and chimney exhausts" studied more cases related to Pressure coefficient fields $C_{p}$ on buildings for various wind directions ; and concluded in the results that areas of over-pressure can be clearly observed on the windward faces $\left(C_{p}>\mathbf{0}\right)$ and areas of negative pressure $\left(C_{p}<0\right)$ on the other faces (leeward faces, roof) based on a $\mathrm{n}$ experimental study and RANS simulations using SST k- $\omega$ turbulence model performed on most cases of wind directions.

Castro and A. G. Robins (1977, England) [8] on their study "The flow around a surface-mounted cube in uniform and turbulent streams" studied An experimental investigation of the flow around surface-mounted cubes that confirm the conclusion of Ricciardi, Thomas, Sandrine about distribution of coefficient pressure $C_{p}$ (where $C_{p}<\mathbf{0}$ and $C_{p}>\mathbf{0}$ ) over different regions of cube as will be shown below in the results.

Also Ricciardi, Thomas, Sandrine (2015, France) recommended that the range of $C_{p}$ values obtained for a null air flow in the chimney (null air flow $(\mathbf{V} / \mathbf{U})=0$ ), noted as $C_{p_{\mathbf{V} / \mathbf{U}=\mathbf{0}}}$, characterizes the aspiration capacity of 
the chimney. If this range of $C_{p_{\mathbf{V} / \mathbf{U}=\mathbf{0}}}$ is entirely negative, it indicates that, whatever the wind direction, the wind will create an aspiration in the chimney and then will assist the exhaust of the air. If, for some wind directions, the values of $\mathbf{C} \mathbf{p}_{\mathbf{V} / \mathbf{U}=\mathbf{0}}$ are positive, the wind can cause back-pressure on the top of the chimney (or cube) and then thwart the exhaust of the air. Where $\mathbf{V}$ is air flow from chimney (or from a vent exist in the center of roof cube), and $\mathbf{U}$ is the wind speed at any point around cube.

This study can help us more to identify the range of coefficient pressure $(\mathbf{C p})$ of a solid cube $(\mathbf{V} / \mathbf{U}=\mathbf{0})$. In this case (solid cube), no air flow will be investigated from the roof of cube $(\mathbf{V} / \mathbf{U}=\mathbf{0})$ as will be shown in the results below Fig 5,6,7.

2. Steps of Modeling and setting up of CFD details using Ansys Fluent (14.5) :

2.1 Importing and Scaling the grid (meshing): Actually, the first important step in Ansys Fluent (14.5) is how to generate the best mesh (grid), as will be shown below.

\subsection{Importing and Scaling the grid :}

First, the numerical solver is set to the following specifications:

- Solver type: Pressure based.

- Formulation: Implicit

- Space: 3D

- Time: steady (we want here steady not transient).

- Velocity formulation: Absolute

- Gradient option: Green-Gauss cell based.

\subsection{Selecting the physical model:}

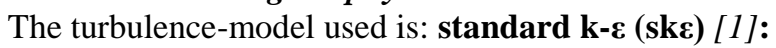

It is a model based on model transport equations for the turbulence kinetic energy $(\mathrm{k})$ and its dissipation rate $(\epsilon)$. The model transport equation for is derived from the exact equation, while the model transport equation for was obtained using physical reasoning and bears little resemblance to its mathematically exact counterpart. In the derivation of the $\mathrm{k}-\epsilon$ model, the assumption is that the flow is fully turbulent, and the effects of molecular viscosity are negligible. The standard k- $\epsilon$ model is therefore valid only for fully turbulent flows as shown:

The turbalance kinetec energy, $k$, and its rate of dissipation, $\varepsilon$, are obtained from the following transport equations:

$$
\frac{\partial}{\partial t}(\rho k)+\frac{\partial}{\partial x_{i}}\left(\rho k u_{i}\right)=\frac{\partial}{\partial x_{j}}\left[\left(\mu+\frac{\mu_{t}}{\sigma_{k}}\right) \frac{\partial k}{\partial x_{j}}\right]+G_{k}+G_{b}-\rho \varepsilon-Y_{M^{+}}+S_{k}
$$

and

$$
\frac{\partial}{\partial t}(\rho \varepsilon)+\frac{\partial}{\partial x_{i}}\left(\rho \varepsilon u_{i}\right)=\frac{\partial}{\partial x_{j}}\left[\left(\mu+\frac{\mu_{t}}{\sigma_{\varepsilon}}\right) \frac{\partial \varepsilon}{\partial x_{j}}\right]+C_{1 \varepsilon} \frac{\varepsilon}{k}\left(G_{k}+C_{3 \varepsilon} G_{b}\right)-C_{2 \varrho} \rho \frac{\varepsilon^{2}}{k}+S_{\varepsilon}
$$

Where:

$\boldsymbol{G}_{\boldsymbol{k}}$ : $\quad$ represents the generation of turbulence kinetic energy due to the mean velocity gradients, calculated as described in Modeling Turbulent Production in the k- $\varepsilon$ Models. 
$\boldsymbol{G}_{\boldsymbol{b}}$ : is the generation of turbulence kinetic energy due to buoyancy, calculated as described in effects of buoyancy on turbulence in the k- $\varepsilon$ Models.

$\boldsymbol{Y}_{\boldsymbol{M}}$ : represents the contribution of the fluctuating dilatation in compressible turbulence to the overall dissipation rate, calculated as described in Effects of Compressibility on Turbulence in the k- $\varepsilon$ Models.

$\boldsymbol{C}_{1 \varepsilon}, \boldsymbol{C}_{\mathbf{2 \varepsilon}}, \boldsymbol{C}_{\mu}$ are constants with following default values are: 1.4, 1.92, and 0.09 respectively, and $\boldsymbol{\sigma}_{\boldsymbol{k}}, \boldsymbol{\sigma}_{\varepsilon}$ with following default values: 1.0, 1.3 respectively. $\boldsymbol{S}_{\boldsymbol{k}}, \boldsymbol{S}_{\boldsymbol{\epsilon}}$ are user-defined source terms. The turbulent (or eddy) viscosity, $\mu_{t}$, is computed by combining $\mathbf{k}$ and $\boldsymbol{\epsilon}$ as follows; where, $\boldsymbol{C}_{\mu}$ is a constant :

$$
\mu_{t}=\rho C_{\mu} \frac{k^{2}}{\varepsilon}
$$

\subsection{Prescribing operating conditions:}

The operating pressure is set to normal atmospheric pressure $(\mathbf{1 0 1 3 2 5} \mathbf{P a})$. The effect of gravity is incorporated in the model by setting the gravity value equal to $\mathbf{- 9 . 8} \mathrm{m} / \mathrm{S}^{2}$ along $\mathbf{y}$-axis.

\subsection{Applying boundary conditions:}

Following boundary conditions are applied to the above-defined zones (Named-selection):

- Setting "velocity inlet" as $0.6 \mathrm{~m} / \mathrm{s}$ with direction $\mathrm{x}$-coordinate (uniform velocity as absolute).

- Setting "outlet pressure" as $0 \mathrm{~Pa}$.

- Setting all sides of cube : as "wall" with neglecting the slip along these walls.

- Set the top side of domain once as "symmetry" and once as "wall".

\subsection{Setting up solver controls:}

The solver controls are set to the following settings:

- Equations: Flow and Energy

- Pressure-velocity coupling: SIMPLE

- Under-Relaxation factors:

- Pressure $=0.3$

- Density = 1

- Body Forces = 1

- Momentum = 0.7

- Turbulence Kinetic Energy $=0.8$

- Discretization:

- Pressure = PRESTO!

- Momentum = Second Order Upwind

- Energy = Second Order Upwind

2.7 Setting up convergence monitors: The convergence monitors are set appropriately and the plotting of residuals (continuity, $\mathbf{x}$-velocity, $\mathbf{y}$-velocity, z-velocity,....etc) during the calculations have been enabled as shown: 


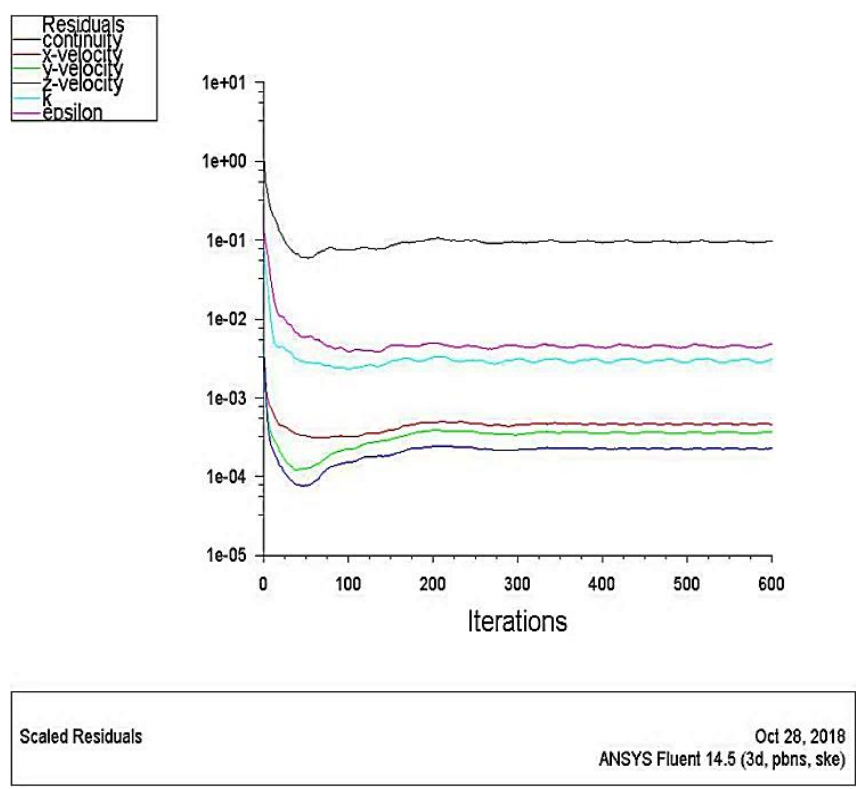

\subsection{Initializing the flow field:}

The calculations were stared by giving the number of iterations with standard initialization from the inlet velocity $\mathbf{0 . 6}(\mathbf{m} / \mathbf{s})$

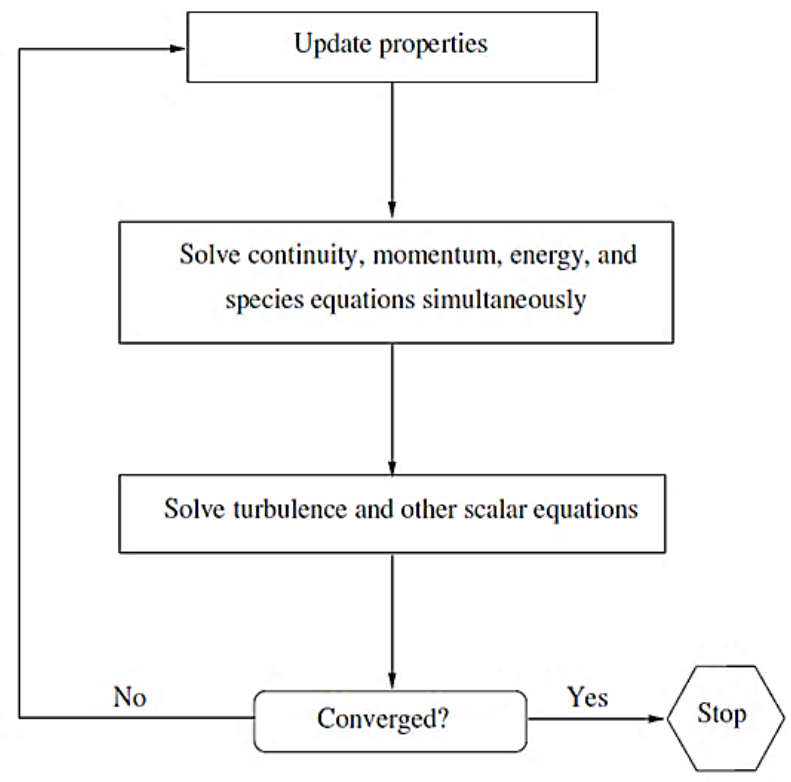

\subsection{Computing and Monitoring the Solution:}

The discretized conservation equations are solved iteratively. A number of iterations are usually required to reach a converged solution as shown above.

Convergence is reached when:

1. Changes in solution variables from one iteration to the next are negligible. Residuals provide mechanism to help monitor this trend.

2. Overall property conservation is achieved. 


\section{Numerical Method}

\subsection{Governing equations:}

The equation for conservation of mass, or continuity equation, can be written as follows:

$$
\frac{\partial \rho}{\partial t}+\nabla \cdot\left(\rho \vec{U}_{i}\right)=S_{m}
$$

This equation is the general form of the mass conservation equation and is valid for incompressible as well as compressible flows. The source $\boldsymbol{S}_{\boldsymbol{m}}$ is the mass added to the continuous phase from the dispersed second phase (e.g., due to vaporization of liquid droplets) and any user-defined sources.

-The mass conversation equation (for steady-state, incompressible, single phase flow) used in this study:

$$
\rho\left(\nabla U_{i}\right)=0
$$

- Conservation of momentum in an inertial (non-accelerating) reference frame is described by :

$$
\frac{\partial}{\partial t}\left(\rho \vec{U}_{i}\right)+\nabla\left(\rho \vec{U}_{j} \vec{U}_{i}\right)=-\nabla P+\nabla \cdot(\bar{\tau})++\rho \vec{g}+\vec{F}
$$

Where $\boldsymbol{P}$ is the static pressure, $\overline{\boldsymbol{\tau}}$ is the stress tensor (described below), and $(\boldsymbol{\rho} \overrightarrow{\boldsymbol{g}})$ and $\overrightarrow{\boldsymbol{F}}$ are the gravitational body force and external body forces (e.g., that arise from interaction with the dispersed phase), respectively. $\vec{F}$ also contains other model-dependent source terms such as porous-media and user-defined sources. The stress tensor $\overline{\boldsymbol{\tau}}$ is given by:

$$
\overline{\boldsymbol{\tau}}=\mu\left[\left(\nabla \vec{U}_{i}+\nabla \vec{U}_{i}^{T}\right)-\frac{2}{3} \nabla \cdot\left(\vec{U}_{i} I\right)\right]
$$

Where $\boldsymbol{\mu}$ is the molecular viscosity, $\boldsymbol{I}$ is the unit tensor, and the second term on the right hand side is the effect of volume dilation.

- The momentum conversation equation(for steady-state, incompressible, single phase flow):

$$
\nabla\left(\rho \vec{U}_{j} \vec{U}_{i}\right)=-\nabla P+\nabla \cdot(\bar{\tau})++\rho \vec{g}
$$

Where $\boldsymbol{i}$ is the index for the $\mathbf{x}, \mathbf{y}, \mathbf{z}$ direction, $\boldsymbol{\rho}\left(\frac{\boldsymbol{k g}}{\boldsymbol{m}^{3}}\right)$ is the density of the fluid, $\boldsymbol{P}$ is the static pressure $\left(\frac{N}{m^{2}}\right), \boldsymbol{\mu}($ $\frac{\boldsymbol{k g}}{\boldsymbol{m} \cdot \boldsymbol{s}}$ ) is the viscosity coefficient, which is sum of the dynamic viscosity $\boldsymbol{\mu}_{\boldsymbol{d}}$ and fluid turbulent viscosity and $\boldsymbol{\mu}_{\boldsymbol{t}}$ $-\boldsymbol{\mu}$ is the total viscosity coefficient:

$$
\mu=\mu_{d}+\mu_{t}
$$

Where, $\mu_{t}=\rho C_{\mu} \frac{k^{2}}{\varepsilon}$ 


\subsection{CFD Setting up of cube closure:}

The computational domain $(14.5 H \times 2 H \times 9 H)$ in $\mathrm{X}, \mathrm{Y}$ and $\mathrm{Z}$ respectively shown in Fig $\mathbf{1}, 2$ and 4 is identical to that used by Mohd ARIFF, Salim M.(2009) [2] , Lakehal and Rodi (1997)[6] in their numerical analysis that replicates the experimental setup of Martinuzzi et al. (1993). A fully developed turbulent flow was set at the inlet and $\boldsymbol{R} \boldsymbol{e}_{\boldsymbol{H}}=\mathbf{4 0 , 0 0 0}$. No-slip conditions were applied on the channel floor, top wall and all cube faces, whereas the side walls were defined as symmetry to reduce computational cost, since they are sufficiently far from the cube to influence the flow characteristics Fig 3.

RANS simulations using Standard $\mathrm{k}-\varepsilon$ turbulence model were performed on all of the study using coarse grid generated using ANSYS 14.5 software with 300,000 (coarse mesh of Y+>33) elements of Geometry as seen in figure 3.

The mean local pressure on a face due to the wind $\boldsymbol{P}_{\boldsymbol{j}}$ is linked to the mean reference dynamic pressure of the wind, noted as $\left(\mathbf{1} / 2 \rho W_{r e f}^{2}\right)$, and the mean pressure coefficient $C_{p_{j}}$ is defined by the following relation (4) and (5):

$C_{p}=\frac{P-P_{a t m}}{1 / 2 \rho W_{r e f}}$

$$
C_{p_{j}}=\frac{P_{j}-P_{o}}{1 / 2 \rho W_{r e f}^{2}}
$$

Where:

$\boldsymbol{P}_{\boldsymbol{j}}$ : mean local pressure due to the wind at a point $\mathrm{j}(\mathrm{Pa})$,

$\boldsymbol{P}_{\mathbf{0}}$ : static pressure far from the building influence zone $(\mathrm{Pa})$,

P : air density (kg/m3),

$\boldsymbol{W}_{\text {ref }}$ : wind reference velocity, measured at reference height (which is here inflow wind velocity) $(\mathrm{m} / \mathrm{s})$.

$\boldsymbol{C}_{\boldsymbol{p}_{\boldsymbol{j}}}$ : mean pressure coefficient at a point $\mathrm{j}$.

(Non dimensionless)

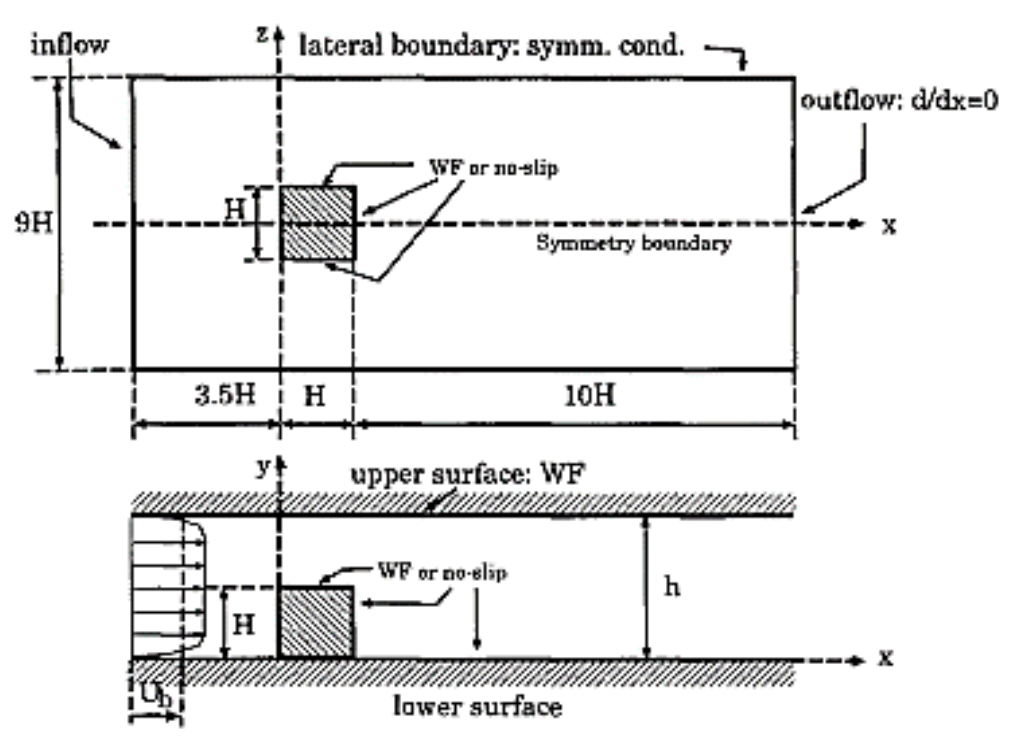

Fig1. Geometry with boundary conditions [6]

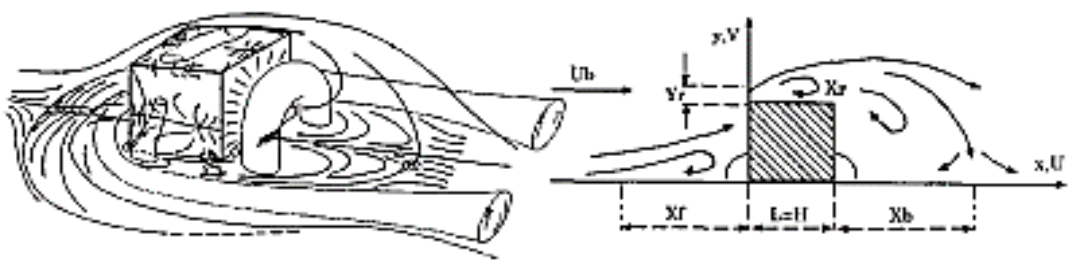

Fig2. Representation of flow around a cube [6] 

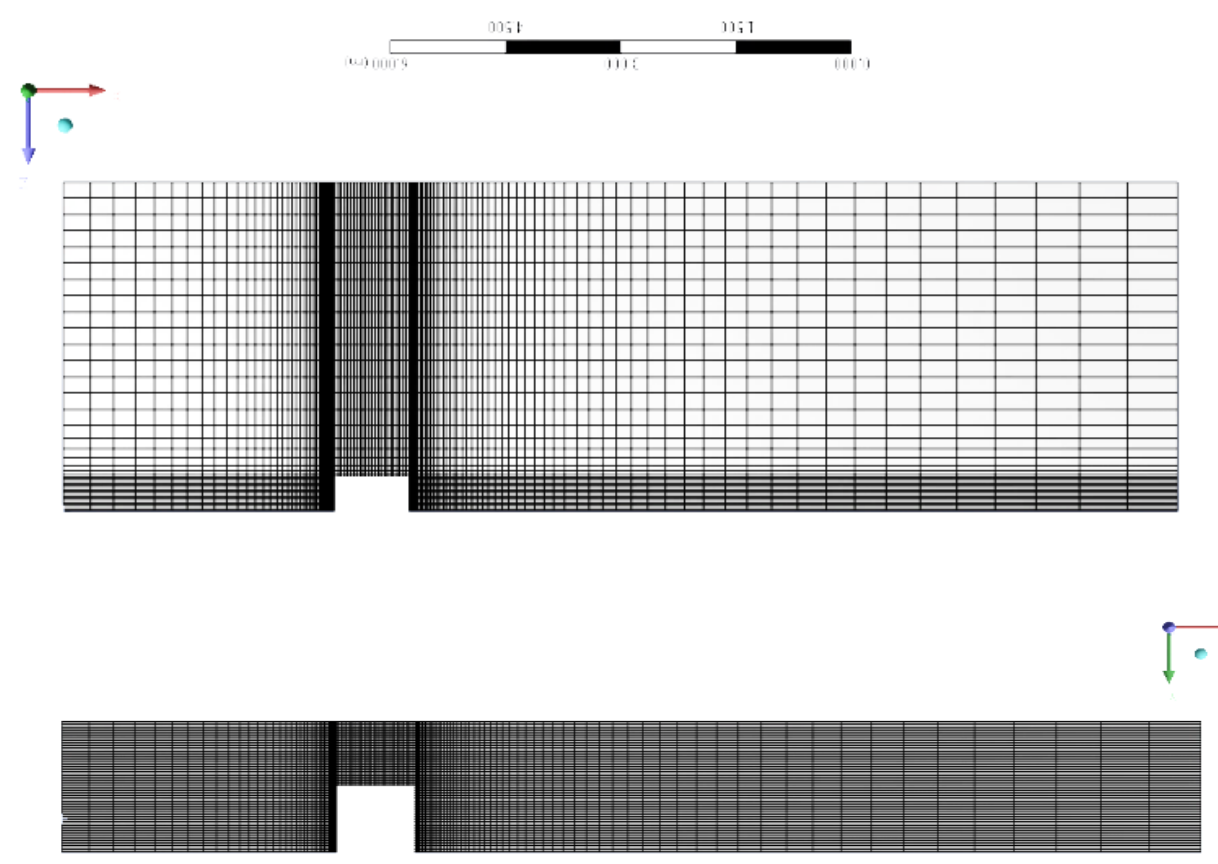

Fig 3. Generated Mesh in Ansys 14.5

Velocity inlet is $0.6 \mathrm{~m} / \mathrm{s}$, pressure outlet is atmospheric pressure put to 0 , Reynold number is almost $\mathbf{4 0 0 0 0}$, the number of elements are about $\mathbf{3 0 0 , 0 0 0}$ elements $(\mathbf{Y}+\mathbf{3 3})$, with neglecting slipping on the sides of walls.

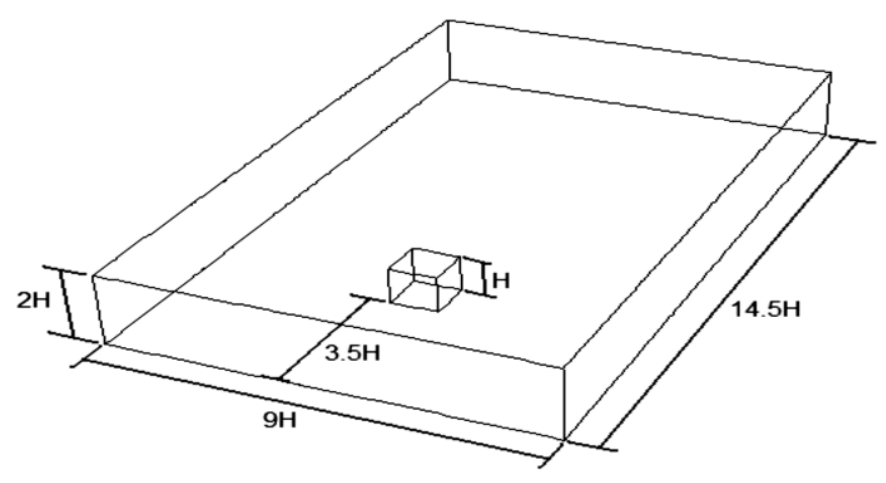

Fig4. Geometry of cube closure 


\section{Results:}
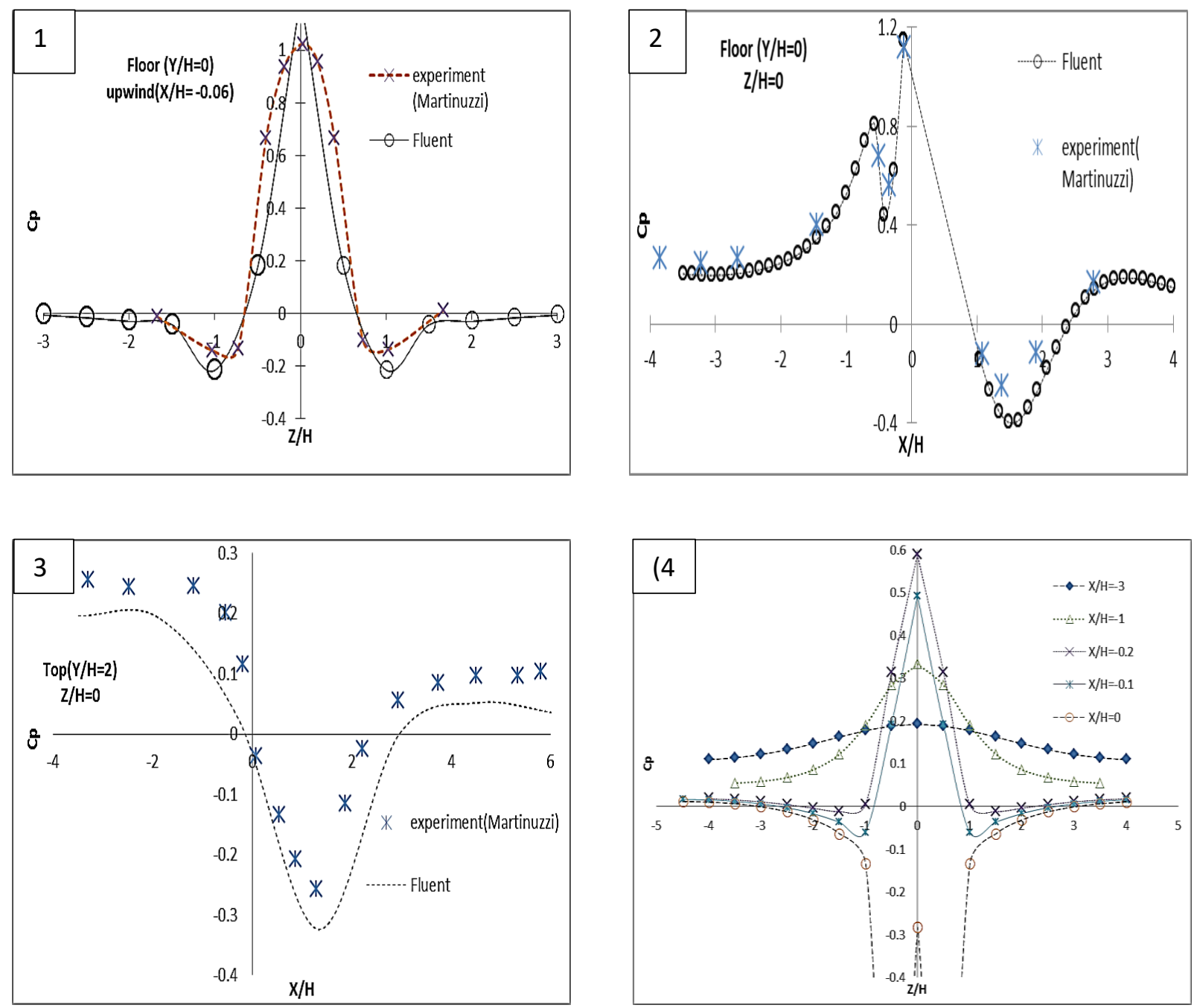

Fig 5. Comparison between the Pressure coefficient of present work and experimental data from Martinuzzi (1993) : ( 1) in plane $\mathrm{Y} / \mathrm{H}=0$ \& Line $\mathrm{X} / \mathrm{H}=-0.06$ (2) in planes $\mathrm{Y} / \mathrm{H}=0$ \& Line $\mathrm{Z} / \mathrm{H}=0$; (3) in planes $\mathrm{Y} / \mathrm{H}=2$ \& $\mathrm{Line} \mathrm{Z} / \mathrm{H}=0$; (4) at Lines : $\mathrm{X} / \mathrm{H}=-3, \mathrm{X} / \mathrm{H}=-1, \mathrm{X} / \mathrm{H}=-0.2, \mathrm{X} / \mathrm{H}=-0.1, \mathrm{X} / \mathrm{H}=0$ on the floor $(\mathrm{Y} / \mathrm{H}=0)$ 


\section{(1)}

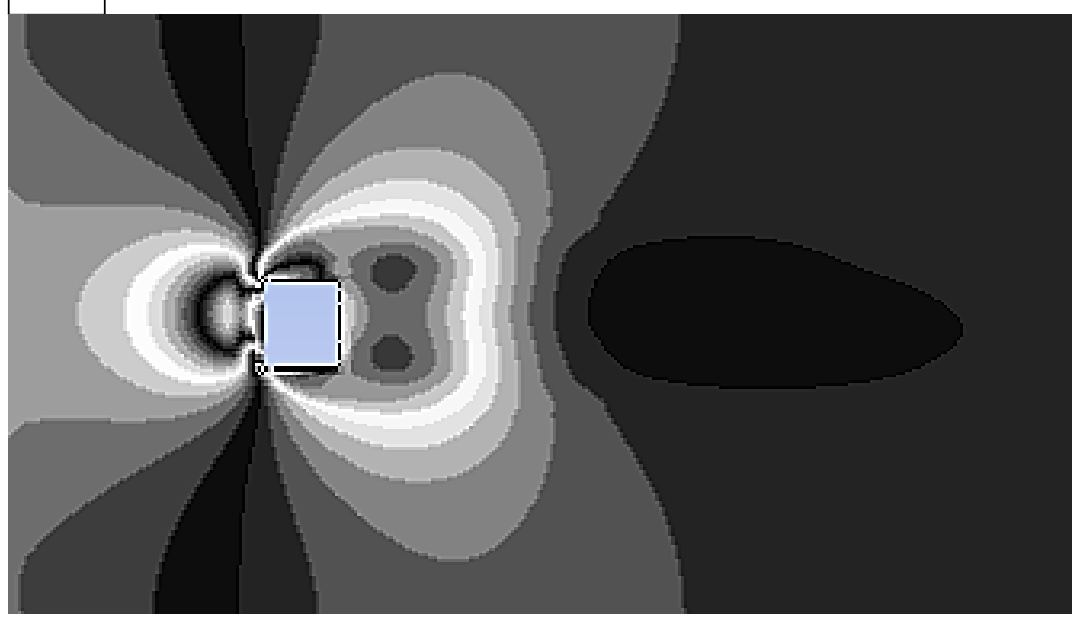

Pressure Coefficient Contour 1

$1.125 \mathrm{e}+000$
$9.660 \mathrm{e}-001$
$8.075 \mathrm{e}-001$
$6.489 \mathrm{e}-001$
$4.903 \mathrm{e}-001$
$=3.318 \mathrm{e}-001$
$-1.732 \mathrm{e}-001$
$1.465 \mathrm{e}-002$
$-1.439 \mathrm{e}-001$
$-3.025 \mathrm{e}-001$
$-4.610 \mathrm{e}-001$
$-6.196 \mathrm{e}-001$
$-7.782 \mathrm{e}-001$
$-9.367 \mathrm{e}-001$
$-1.095 \mathrm{e}+000$
$-1.254 \mathrm{e}+000$
$-1.412 \mathrm{e}+000$

(2)

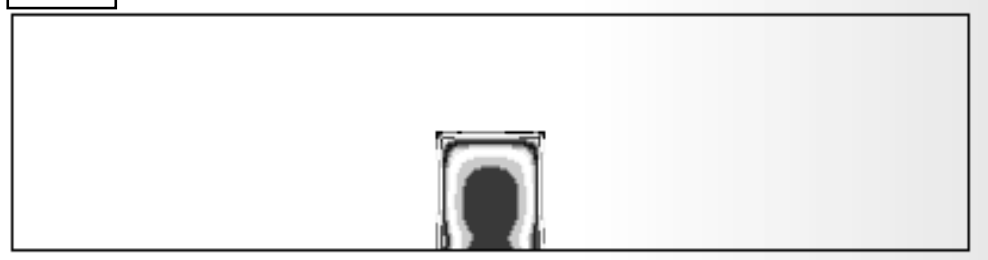

Pressure Coefficient

$\square 1.177 \mathrm{e}+000$

9.616e-001

$7.458 \mathrm{e}-001$

5.300e-001

3.142e-001

9.834 e-002

-1.175 e-001

-3.333e-001

-5.491 e-001

$-7.650 e-001$

$-9.808 e_{-001}$

$-1.197 \mathrm{e}+000$

$-1.412 \mathrm{e}+000$

\section{(3)}

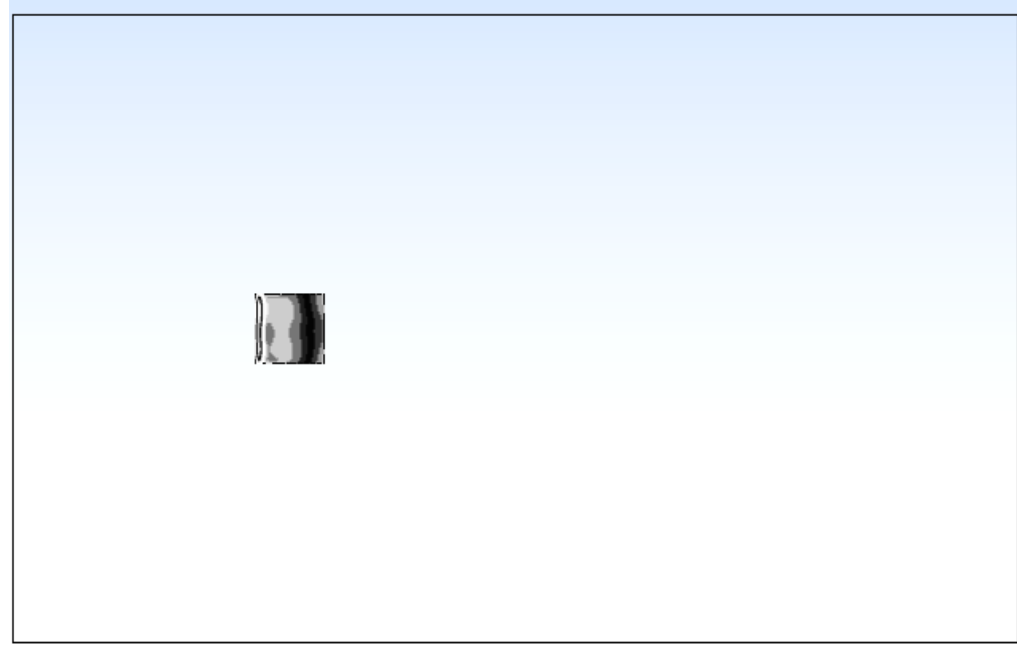

Pressure Coefficier Contour 1

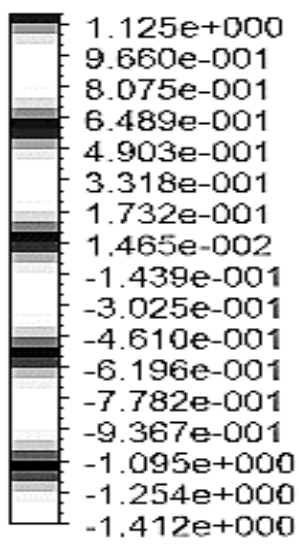

Fig 6. Coefficient pressure contours on: (1) the floor plane $(\mathrm{Y} / \mathrm{H}=0)$. (2) the upwind side of cube $(\mathrm{X} / \mathrm{H}=0)$. (3) the top side of cube 


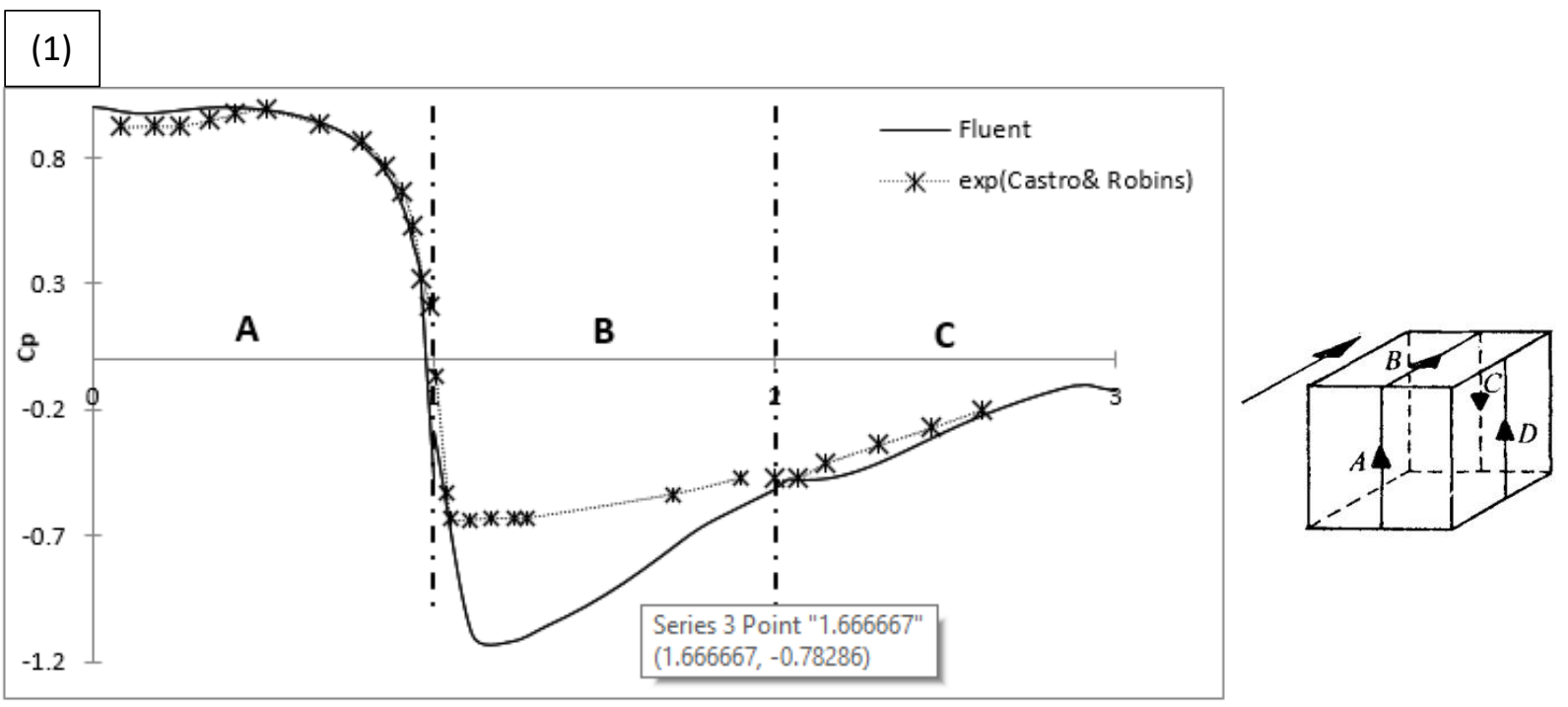

$(2)$
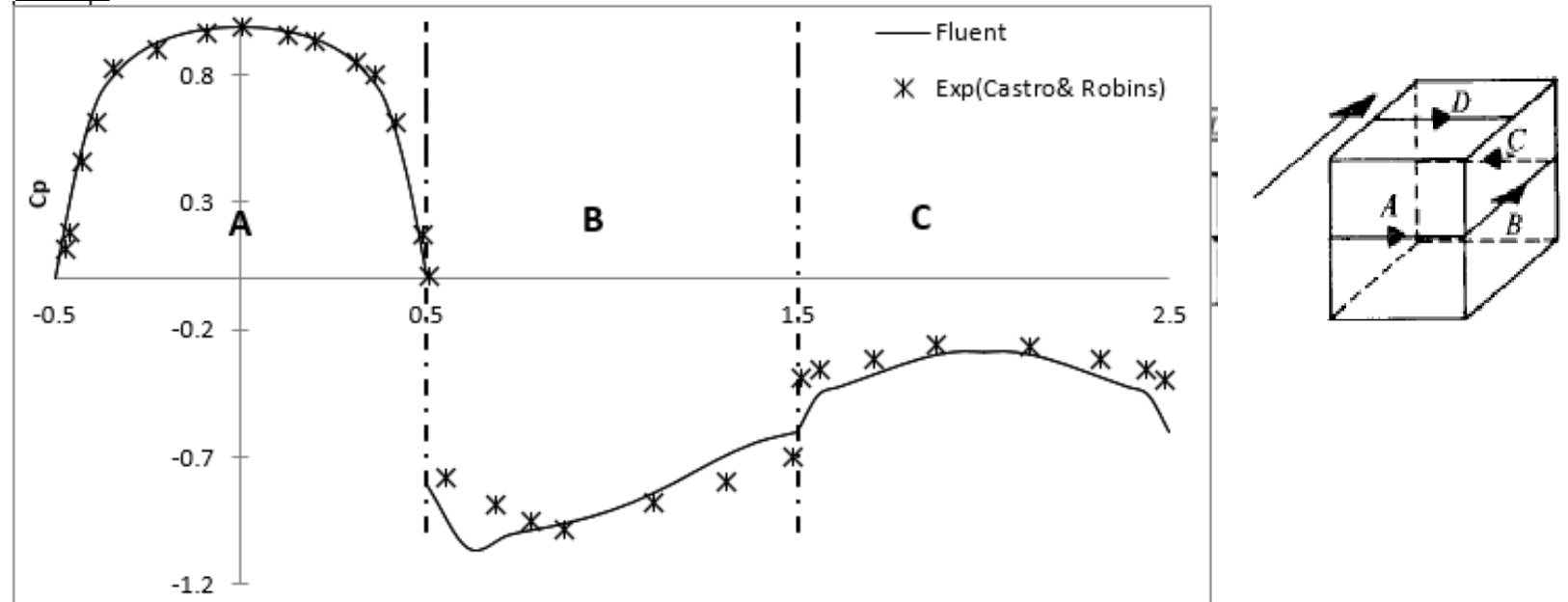

Fig7. Coefficient pressure on the different sides of cube closure with different directions as shown in (1) longitudinal direction (2) lateral direction with a comparison from Castro \& Robins experiment. 


\section{Discussion and Conclusion:}

All the results of this study are based on Steady RANS simulation in ANSYS Fluent 14.5 and we have got a good acceptance with experimental works. Fig5 represent a comparison between the Pressure coefficient of present work and experimental data from Martinuzzi on the floor and top of cube; where Fig 5.1 illustrates the behavior of coefficient pressure around the cube on the floor along the lateral (z-axis) with range (-0.2:1), the maximum value of $\boldsymbol{C}_{\boldsymbol{p}}$ occurs at center line of cube $(\mathbf{Z}=\mathbf{0})$ on the floor, the minimum occurs in the symmetrical sides of geometry at points $(\mathbf{Z}=\mathbf{- 1} \& \mathbf{Z}=\mathbf{1})$. Fig $\mathbf{5 . 2}$ illustrates the behavior of coefficient pressure around the cube on the floor along the horizontal (x-axis) at center line of cube with range (-0.4:1.1), the maximum value of $\boldsymbol{C}_{\boldsymbol{p}}$ occurs at a point before $(\mathbf{x}=\mathbf{0})$ from the upwind side on the floor.

Fig 5.3 illustrates the behavior of coefficient pressure around the cube on the top of cube along the horizontal center line (x-axis) with range (-0.3:0.2), the minimum value of $\boldsymbol{C}_{\boldsymbol{p}}$ occurs at point before $(\mathbf{X}=\mathbf{1})$ of value $\mathbf{0 . 3}$, and the fig $\mathbf{5 . 4}$ describes the behavior of $\boldsymbol{C}_{\boldsymbol{p}}$ on the floor along the lateral ( $\mathbf{Z}$-axis); how it is changing with increasing the maximum point of $\boldsymbol{C}_{\boldsymbol{p}}$ at $\mathbf{Z}=\mathbf{0}$ in the upwind region gradually from the farther upwind distance line $(\mathbf{X}=\mathbf{- 3})$ approaching to the line distance $(\mathbf{X}=\mathbf{0})$ with sudden change of high negative value of $\boldsymbol{C}_{\boldsymbol{p}}$. Contours of $\boldsymbol{C}_{\boldsymbol{p}}$ on: (1) the floor in plane (Y/H=0). (2) the upwind side of cube at plane. $(\mathrm{X} / \mathrm{H}=0)$. (3) the top side of cube show the behavior of pressure coefficient changes. (fig 6.1, fig 6.2 and fig 6.3 respectively).

Due to presence the cube in the domain of wind tunnel, velocity of wind will be affected to perform the reverse flow near the roof of the cube as shown in fig2; where velocity profiles start to change near the cube forming the shown behavior along the horizontal direction.

Also, we can notice that the range of coefficient Pressure $\boldsymbol{C}_{\boldsymbol{p}}$ on the face $\mathbf{A}$ with directions as shown in fig 7.1 and fig 7.2 is entirely positive $\left(\boldsymbol{C}_{\boldsymbol{p}}>0\right)$, Also, the range of coefficient Pressure $\boldsymbol{C}_{\boldsymbol{p}}$ on the faces B,C of solid cube in the different directions is entirely negative $\left(\boldsymbol{C}_{\boldsymbol{p}}<0\right)$. As shown in fig 7.1 and fig 7.2.

\section{Nomenclature:}

H - Height of Obstacle (m).

$\mathbf{Z}$-crosswind direction $(\mathrm{m})$.

$\mathbf{X}$ - Horizontal Distance along Stream wise direction $(\mathrm{m})$.

$\mathbf{Y}$ - Vertical Distance normal to the ground direction.

$\boldsymbol{u}_{\boldsymbol{B}}-$ Bulk Velocity $(\mathrm{m} / \mathrm{s})$.

XR - Reattachment Length $(\mathrm{H})$.

$\mathbf{y + - D i m e n s i o n l e s s ~ d i s t a n c e ~ t o ~ t h e ~ w a l l ~ . ~}$
$\boldsymbol{R e}_{\boldsymbol{H}^{-}}$Reynolds Number $\left(=\left(\mathrm{H}^{*} u_{B}\right) / v_{\text {air }}\right)$.

$\mathbf{U}$ - Instantaneous Velocity $(\mathrm{m} / \mathrm{s})$.

XF - Frontal Separation length $(\mathrm{H})$.

k - Turbulent kinetic energy $\left(\mathrm{m}^{2} / \mathrm{s}^{2}\right)$. $\boldsymbol{u}_{\boldsymbol{\tau}}$ - Friction Velocity $(\mathrm{m} / \mathrm{s})$.

$\boldsymbol{\varepsilon}$ - Dissipation rate $\left(\mathrm{m}^{2} / \mathrm{s}^{2}\right)$.

$\boldsymbol{v}_{\text {air }}$-Kinematic viscosity of $\operatorname{air}\left(\mathrm{m}^{2} / \mathrm{S}\right)$. 


\section{References :}

[1]B. E. Launder and D. B. Spalding. Lectures in Mathematical Models of Turbulence. Academic Press, London, England. 1972.

[2]. ARIFF, M., SALIM, S.M., and CHEAH. "WALL Y+ APPROACH FOR DEALING WITH TURBULENT FLOW OVER A SURFACE MOUNTED CUBE”: PART 2 - HIGH REYNOLDS NUMBER”(2009)

[3] HUSSEIN, H.J., and MARTINUZZI, R.J., (1996), "Energy Balance for Turbulent Flow around a Surf Mounted Cube Placed in a Channel”, Phys. Fluids 8 (3), 764-780.

[4] S. A. Orszag, V. Yakhot, W. S. Flannery, F. Boysan, D. Choudhury, J. Maruzewski, and B. Patel. "Renormalization Group Modeling and Turbulence Simulations". In International Conference on NearTurbulent Flows Tempe, Arizona.1993.Wall

[5] FLUENT (14.5), Documentation: User guide, ANSYS Inc.

[3]MARTINUZZI, R., and TROPEA, C., (1993), “The Flow around Surface Mounted Prismatic Obstacles Placed in a Fully Developed Channel Flow”, Trans ASME J. Fluid Eng 115 (1) 85-91.

[6] LKEHAL, D., and RODI, W., (1997), "Calculation of the flow past a surface of a mounted cube with 2-layer turbulence model”, J. Wind Eng. and Ind. Aerodynamics. 67-68, 66-78.

[7] Yoshihide Tominaga, Ted Stathopoulos (2009) “Numerical simulation of dispersion around an isolated cubi building: Comparison various types of $\mathrm{k}-3$ models".

[8] Castro and A. G. Robins (1977, England) “The flow around a surface mounted cube in uniform and turbulent streams"

[9] Ricciardi, Thomas, Sandrine (2015, France) : " Experimental and numerical characterization of windinduced pressure coefficients on nuclear buildings and chimney exhausts" 